Écrire

l'histoire

\section{Écrire l'histoire}

Histoire, Littérature, Esthétique

$10 \mid 2012$

Mensonges (2)

\title{
Le mensonge en histoire
}

Le télégramme $\mathrm{n}^{\circ} 118$ du Livre jaune (décembre 1914)

Jean-Jacques Becker

\section{(2) OpenEdition}

Journals

Édition électronique

URL : http://journals.openedition.org/elh/196

DOI : 10.4000/elh.196

ISSN : 2492-7457

Éditeur

CNRS Éditions

\section{Édition imprimée}

Date de publication : 18 décembre 2012

Pagination : $37-45$

ISBN : 978-2-35698-050-2

ISSN : 1967-7499

Référence électronique

Jean-Jacques Becker, «Le mensonge en histoire », Écrire l'histoire [En ligne], 10 | 2012, mis en ligne le

18 décembre 2015, consulté le 23 septembre 2020. URL : http://journals.openedition.org/elh/196 ;

DOI : https://doi.org/10.4000/elh.196 


\section{Le mensonge en histoire Le télégramme $n^{\circ} 118$ du Livre jaune (décembre 1914)}

LE mensonge, Le Demi-Mensonge, l'altération de la vérité sont extrêmement fréquents aussi bien dans la vie politique intérieure que dans les relations internationales, mais ces offenses à la vérité ont souvent peu de conséquences, ou du moins des conséquences assez limitées. Une question est posée ici: les mensonges du Livre jaune français en 1914 ont-ils changé le cours de l'histoire, ou même l'ont-ils seulement accompagné?

Rappelons les faits.

Les dépêches reçues par les ministères des Affaires étrangères des différents pays concernés au moment du déclenchement de la Grande Guerre ont été réunies dans les mois qui ont suivi dans des livres dits de « couleur », nommés d'après leur couverture : livre jaune pour la France, orange pour la Russie, rouge pour l'Autriche-Hongrie, bleu pour la Serbie et également pour le RoyaumeUni, blanc pour l'Allemagne, gris pour la Belgique. Disons-le immédiatement, ces ouvrages n'ont pas été des modèles de travail scientifique. Ce sont en réalité des ouvrages de propagande; dans chaque pays, on a publié certains documents et laissé d'autres de côté, quand on n'en a pas transformé le contenu en fonction de la démonstration qu'ils devaient servir: essentiellement, que c'était aux autres que revenait la responsabilité de la guerre. En France, le Livre jaune - son titre exact est Documents diplomatiques. 1914, La Guerre européennea été établi sous la direction de Philippe Berthelot, secrétaire général du Quai d'Orsay ${ }^{1}$, et publié en décembre 1914.

1. Philippe Berthelot (1866-1934), fils du chimiste Marcellin Berthelot, est l'un des principaux diplomates français de cette période. En tant que secrétaire général du ministère des Affaires étrangères, il est l’âme de la politique étrangère pendant la guerre. 
Outre les manipulations délibérées que nous venons d'évoquer, les dépêches pouvaient contenir des erreurs involontaires.

Le grand historien belge Jean Stengers (19222002), qui a beaucoup travaillé sur ces questions, concluait son étude sur l'entrevue de l'empereur Guillaume II et du roi Albert à Potsdam, en novembre 1913, par cette interrogation:

Dans combien d'autres cas, dans les sources qu'il a à sa disposition, ['historien] n'en est-il pas réduit au produit final, sans pouvoir prendre la mesure de ce qu'a été le processus éventuel de déformation ${ }^{2}$ ? ».

Sans reprendre dans le détail les éléments de l'article que nous avions publié dans Matériaux pour l'histoire de notre temps ${ }^{3}$, rappelons que, lorsque l'empereur allemand et le roi des Belges s'étaient rencontrés à Potsdam en novembre 1913, Guillaume II avait fait part au roi Albert de son inquiétude devant l'attitude de la France, dont l'agressivité envers l'Allemagne rendait une guerre inévitable. Or la dépêche de Jules Cambon, alors ambassadeur de France à Berlin, relative à cette entrevue, avait été complètement récrite par les services du Quai d'Orsay pour lui faire dire exactement le contraire: aux termes de la version publiée, c'était l'Allemagne qui voulait la guerre. Cette réécriture avait pour but de montrer la préméditation allemande dans le déclenchement du grand conflit européen. Si l'on ajoute que Jean
Stengers avait établi que déjà la dépêche de Jules Cambon altérait les propos de l'empereur, et qu'il n'a pas réussi à savoir pourquoi l'ambassadeur de France avait agi ainsi, on conçoit l'ampleur des déformations. Certes, Guillaume II se trompait ou était mal informé quand il affirmait que « la France voulait la guerre »; mais tel n'est pas ici l'objet du débat. Au demeurant, tout cela n'eut aucune importance sur le moment: la dépêche ne fut exhumée que plus tard, pour être ajoutée aux arguments justifiant l'article 231 du traité de Versailles, qui faisait endosser à l'Allemagne et à ses alliés la totale responsabilité de la guerre.

Mais d'autres faux, ou de simples erreurs - il est difficile quelquefois de faire la différence -, ont pu avoir de graves conséquences. Dans ce domaine, une des grandes interrogations est de savoir qui a « commencé » en 1914.

Bien sûr, à l'heure actuelle, on le sait.

À la suite de l'attentat de Sarajevo, le 28 juin 1914, et de la mort de l'archiduc héritier d'Autriche-Hongrie, François-Ferdinand, les réactions d'une grande partie de l'opinion avaient été très violentes dans la double monarchie. Les responsables étaient de toute évidence les Serbes et la Serbie. En réalité, l'enquête qui fut menée alors du côté autrichien établit que, si les conjurés avaient incontestablement trouvé des complicités en Serbie, le gouvernement serbe n’y était pour

2. Jean Stengers, « Guillaume II et le roi Albert à Potsdam en novembre 1913 », Bulletin de la classe des lettres et des sciences morales et politiques de l'Académie royale de Belgique, 7 déc. 1993, p. 253.

3. Jean-Jacques Becker, « Le secret et le faux », Matériaux pour l'histoire de notre temps, n 58, avr.-juin 2000, p. 33-38. 
rien. Toutefois, le ministre des Affaires étrangères austro-hongrois, le comte Berchtold, et le chef d'état-major de l'armée, Conrad von Hötzendorf, jugèrent que c'était là une bonne occasion de mater la Serbie, qui, à leurs yeux, représentait un danger pour l'Empire. L'empereur François-Joseph, ennemi des aventures, mais très touché par ce que représentait l'assassinat de son héritier - même s'il ne l'aimait guère...--, se laissa difficilement convaincre; il estima cependant qu'en aucun cas l'Autriche-Hongrie ne pouvait agir sans avoir consulté l'Allemagne. Or Guillaume II, dont l'archiduc assassiné était un ami personnel, et son chancelier Bethmann-Hollweg pensèrent qu'il fallait laisser agir l'Autriche-Hongrie contre la Serbie ; même si cela conduisait à un conflit armé, celui-ci resterait localisé.

Le 23 juillet à 18 heures, le gouvernement austro-hongrois faisait remettre au gouvernement serbe un ultimatum dont les termes, en réalité, mettaient gravement en cause l'indépendance de la Serbie. La Serbie, du moins en apparence, l'accepta néanmoins très largement, au point que Guillaume II considéra que l'AutricheHongrie avait, sans tirer un coup de feu, obtenu un grand succès. Cela parut toutefois insuffisant au gouvernement austro-hongrois. Le 28 juillet, dans la matinée, l'Autriche-Hongrie déclarait la guerre à la Serbie. L'heure de la remise de l'ultimatum à la Serbie avait été choisie de façon que les présidents de la République et du Conseil français, Raymond Poincaré et René Viviani, soient alors en mer, sur le bateau qui les ramenait du séjour qu'ils venaient d'effectuer en Russie du 21 au 23 juillet: on avait pensé à Vienne que la difficulté pour les deux alliés de se concerter serait un autre élément favorable à une localisation du conflit.

Dans la pratique, ce fut exactement le contraire, car l'attitude de la Russie fut très différente de celle que l'on avait imaginée. Tant le gouvernement que l'opinion - qui, du moins celle des villes, semble avoir joué un grand rôle - considérèrent l'attitude austro-hongroise comme inacceptable. Réuni le 24 juillet dans l'après-midi, le Conseil des ministres russe avait réagi à l'ultimatum en affirmant à l'unanimité que «l'honneur de la Russie, sa dignité, sa mission historique, si elle veut conserver son rang en Europe, exigent qu'elle soutienne la Serbie, et, s'il le faut, par les armes ».

Mais cette déclaration, d'ailleurs restée secrète, ne précisait pas comment elle devait se traduire dans les faits. La grande idée fut d'opérer une mobilisation partielle clairement dirigée contre l'Autriche-Hongrie afin que l'Allemagne ne se sente pas menacée et reste en dehors de la crise; mais les généraux russes estimèrent que c'était techniquement impossible, et finalement, après avoir longtemps résisté, l'empereur Nicolas II acceptait, le 30 juillet dans l'après-midi, que soit lancé l'ordre de mobilisation générale.

À ce moment, aucun autre pays ne l'avait encore fait. Le premier pays à suivre l'exemple 
russe fut l'Autriche-Hongrie, où la mobilisation générale était décrétée le 31 juillet à 12 h 23 .

Dès lors, les militaires jouèrent dans les différents pays un rôle essentiel. La plupart étaient convaincus qu'une guerre qui reposait sur une mobilisation générale, qui mettait donc en ligne un nombre d'hommes considérable, pourvus de moyens techniques très importants, ne pourrait qu'être brève, et que les armées prêtes les premières à entrer en action disposeraient d'un avantage majeur, peut-être décisif, d'où l'importance de ne pas prendre fût-ce vingt-quatre heures de retard sur l'adversaire. C'était d'ailleurs également l'une des raisons de la mobilisation russe, qui, compte tenu de l'immensité du pays, était nécessairement plus lente qu'ailleurs.

Comme le soulignent le général Gambiez et le colonel Suire dans leur Histoire de la première guerre mondiale, «à partir de cette date [30 juillet], tout cède devant les exigences des états-majors qui craignent d'être pris de court ${ }^{4}{ }$. Or pour le commandement allemand (le général Moltke, le neveu du vainqueur de 1870), une guerre contre la Russie devait être en même temps une guerre contre la France, son alliée. Le plan Schlieffen, dont la dernière version datait de 1905 et que Moltke avait légèrement remanié, prévoyait de profiter de la lenteur probable de la mobilisation russe pour accabler l'armée française en quelques jours, avant de pouvoir se retourner contre la Russie. Il ne fallait donc pas perdre de temps. Le $1^{\mathrm{er}}$ août, quand elle eut la certitude que la mobilisation russe était effective - les diplomates allemands en poste en Russie avaient pu confirmer que les grandes affiches rouges de la mobilisation étaient bien apposées sur les murs et, pour qu'il n'y ait pas d'erreur possible, le commandement avait envoyé des informateurs au-delà de la frontière germanorusse pour s'en assurer -, l'Allemagne mobilisait et le même jour, après le refus de la Russie d'arrêter sa mobilisation, lui déclarait la guerre. Du côté français, le même jour également, pratiquement à la même heure - 16 heures -, le commandement avait arraché l'ordre de mobilisation au pouvoir civil. À 8 heures du matin, le général Joffre avait envoyé ce message au gouvernement:

Je vous répète ce que je vous ai dit hier: si le gouvernement tarde à donner l'ordre de mobilisation générale, il m'est impossible de continuer à assumer la responsabilité des hautes fonctions dont sa confiance m'a investies.

Il est donc clair que le signal de la mêlée générale avait été donné par la mobilisation russe.

Jules Isaac, que la librairie Hachette appela après la guerre à reprendre la direction de la plus célèbre collection de manuels du secondaire elle devint alors la collection « Malet-Isaac », en hommage à son premier directeur, Albert Malet, qui s'était engagé à cinquante ans et avait été tué

4. Maurice Suire, Fernand Gambiez, Histoire de la première guerre mondiale, Fayard, 1968, t. 1, Crépuscule sur l'Europe, p. 84. 
en Artois en 1915 -, publiait en 1933 Un débat historique, 1914. Le problème des origines de la guerre ${ }^{5}$. Il s'y interrogeait:

Aurait-on évité la guerre si l'ordre de mobilisation générale n'avait pas été lancé le 30 juillet? Très probablement non. La mobilisation générale russe rendait-elle la guerre inévitable? Certainement oui.

Rappelons néanmoins cette autre affirmation du plus grand historien de cette époque, Pierre Renouvin, qui estimait dans Les Origines immédiates de la guerre ${ }^{6}$ qu'une guerre retardée était peutêtre une guerre évitée et que, sans l'attentat de Sarajevo, la guerre n'aurait pas nécessairement eu lieu; les mobilisations autrichienne et russe n'en étaient que la conséquence.

Comme nous le remarquions dans L'Année 14 :

Même quand le commandement allemand a été convaincu de la nécessité de la guerre, il n’en était pas ou plus de même du pouvoir civil. Les militaires n'auraient pas pu l'emporter sans la mobilisation russe. Ils n'auraient pu « bousculer » le pouvoir civil. Quand on sait dans quelles conditions les militaires russes ont arraché l'ordre de mobilisation au Tsar, on se dit que le pire a tenu à peu de chose. ${ }^{7}$

En résumé, sans la mobilisation russe, la guerre aurait probablement eu tout de même lieu, mais ce n'est pas sûr. En fait, personne, ni à l'époque ni plus tard, ne peut dire ce qui se serait passé.

\section{Et c'est là que le Livre jaune entre en ligne!}

Il y a beaucoup d'incertitude sur le moment où le gouvernement français a connu la mobilisation générale russe. Le rôle de l'ambassadeur français Maurice Paléologue dans ces instants est assez obscur. Il a d'un côté très largement dépassé ses instructions en faisant savoir au gouvernement russe, le 28 juillet, que la Russie avait le plein appui de la France, même si l'on peut considérer qu'il ne faisait que reprendre l'esprit des vigoureux discours prononcés par Raymond Poincaré avant de quitter la Russie. D’un autre côté, il n'a évidemment été aucunement invité à participer aux délibérations des dirigeants russes ni particulièrement tenu au courant de leur déroulement. Le mystère réside dans le moment où le gouvernement français a été informé de la mobilisation générale russe. Quelles qu'aient été ses raisons, Paléologue ne s'est pas précipité puisque sa dépêche n'est partie, via Bergen en Norvège, que le 31 juillet à $10 \mathrm{~h} 43$, alors que la mobilisation générale russe datait du 30 juillet dans l'après-midi. Là où les choses se corsent, c'est que cette dépêche n'est arrivée au ministère français des Affaires étrangères qu'à 20 h 30, et que l'on n'a jamais su les raisons de ce retard! L'historien italien Luigi Albertini est persuadé que, par un mélange de dissimulation et d'in-

5. Jules Isaac, Un débat historique, 1914. Le problème des origines de la guerre, Rieder, 1933.

6. Pierre Renouvin, Les Origines immédiates de la guerre (28 juin-4 août 1914), A. Costes, 1925.

7. Jean-Jacques Becker, L'Année 14, A. Colin, 2004, p. 147.

Jean-Jacques Becker, «Le mensonge en histoire. Le télégramme n 118 du Livre jaune (décembre 1914)» Écrire l'histoire, $\mathrm{n}^{\circ} 10$ - automne 2012, 37-45 
formations tardives, l'ambassadeur Paléologue a sciemment maintenu Paris dans le «noir » sur la mobilisation russe ${ }^{8}$. En outre, lorsque le Conseil des ministres français se réunit ce 31 juillet à partir de 21 heures, l'information se télescope pratiquement avec l'annonce de l'assassinat de Jean Jaurès, perpétré ce même 31 juillet à $21 \mathrm{~h} 30$.

Pour des raisons très diverses, le gouvernement français se trouve donc assez largement « hors jeu » au moment où l'histoire du monde était en train de se jouer.

On peut penser que les ministres français, finalement, n'ont pas su quand a eu lieu exactement la mobilisation générale russe. Dans ces heures fiévreuses, messages diplomatiques et communiqués des agences de presse se croisent dans un ordre indéterminé, de jour et de nuit; les dirigeants, aux prises avec des décisions d'une incroyable gravité, surmenés et privés de sommeil, participent à d'innombrables réunions et sont de plus confrontés à des décalages horaires en matière d'information.

En revanche, plusieurs mois plus tard, c'est sciemment qu'a été prise la décision d'affirmer dans le Livrejaune que la mobilisation autrichienne avait précédé la mobilisation russe et que celle-ci avait été une simple réplique.

Même retardée, comme nous l'avons vu, la dépêche de Maurice Paléologue disait simplement: «La mobilisation générale de l'armée russe a été ordonnée. » Dans le Liore jaune, a été substitué un autre texte, sous le numéro 118 , à la page 116 :

M. Paléologue, Ambassadeur de France à Saint-Pétersbourg, à M. René Viviani, Président du Conseil, Ministre des Affaires étrangères.

Saint-Pétersbourg, le 31 juillet 1914.

En raison de la mobilisation générale de l'Autriche et des mesures de mobilisation prises secrètement, mais d'une manière continue, par l'Allemagne depuis six jours, l'ordre de mobilisation générale de l'armée russe a été donné, la Russie ne pouvant, sans le plus grave danger, se laisser davantage devancer; en réalité, elle ne fait que prendre des mesures militaires correspondant à celles prises par l'Allemagne.

Pour des raisons stratégiques impérieuses, le Gouvernement russe ne pouvait plus, sachant que l'Allemagne s'armait, retarder la conversion de sa mobilisation partielle en mobilisation générale.

Ce texte a donc été totalement fabriqué par des fonctionnaires du Quai d'Orsay. Pratiquement tout y est faux.

Compte tenu de la date de publication du Livre jaune, en décembre 1914, on peut s’interroger sur l'intérêt d'une telle «fabrication ». Les hostilités avaient commencé depuis près de cinq mois. L’armée allemande, passant par la Belgique, avait

8. Luigi Albertini, The origins of the war of 1914, Westport (Conn.), Greenwood Press, 1980, t. II, p. 323-325; traduction en anglais établie par Isabella M. Massey de Le origini della guerra del 1914, Milan, Bocca, 1942-1943. 
envahi la France du Nord et, sans la victoire de la Marne, considérée comme presque miraculeuse, l'Allemagne aurait déjà gagné la guerre, sur le front occidental du moins. Certes, on n'imaginait pas que le conflit durerait encore quatre années, mais il était déjà évident qu'il serait beaucoup plus long que prévu et que, dans cette mêlée des peuples d'une ampleur inconnue, la propagande prenait également une place inconnue jusqu'alors.

Il n'était peut-être pas sans importance de réaffirmer pour l'opinion française que la France avait été victime d'une agression, mais il n'est pas très difficile de se figurer qu'en ce mois de décembre 1914 ils ne furent pas très nombreux, combattants ou non-combattants, à lire le Livre jaune, ni même les organes de presse à en faire état. Il fallait surtout le proclamer à la face $\mathrm{du}$ monde. Bien avant la publication du Livre jaune, l'opinion française avait été convaincue de l'agression allemande. Un homme comme Clemenceau, pour ne citer que cet exemple, en a été persuadé non seulement dès le début du conflit et durant toute la guerre, mais jusqu'à sa mort en... 1929. Sans même volonté de truquer l'histoire, la façon dont les journaux avaient rendu compte des événements avait nécessairement été empreinte d'un grand flou. Nulle part ne sont mentionnées dans un journal comme Le Temps les dates des mobilisations autrichienne et russe; quant au Matin, journal de grande diffusion, il écrit le samedi $1^{\mathrm{er}}$ août, sans paraître attacher une grande importance aux dates: «La mobilisation est devenue générale, hier matin en Autriche, hier soir en Russie. » Même l'ambassadeur de Russie en France, Alexandre Isvolsky, ne savait pas exactement ce qu'il en était.

Après la révolution de 1917, les bolcheviks ont ouvert les archives diplomatiques de l'époque tsariste, qui ont été traduites dans les années vingt sous le titre Un livre noir. Diplomatie d'avant-guerre d'après les documents des archives russes et publiées en France par la Librairie du Travail, une maison d'édition communiste. Dans le deuxième tome, qui s'étend de novembre 1910 à juillet 1914, on trouve ce télégramme d'Isvolsky, télégramme no 214 daté des 18/31 juillet ${ }^{9}$ qui se termine de la façon suivante: Il est remarquable que dans le télégramme Havas, de Berlin, reçu ici, il est parlé de la «mobilisation générale de l'armée russe », ce qui donne à penser que l'Allemagne s'efforce de provoquer prématurément dans tel ou tel autre but une mesure analogue de la part de la France.

Aussi étonnant que cela puisse paraître, l'ambassadeur russe en France ne savait pas que la mobilisation générale avait été décrétée dans le pays qu'il représentait!

De sorte que, si le Liore jaune n'allait évidemment être d'aucun effet sur l'ensemble de la population, il joua en revanche un grand rôle dans l'analyse des événements par ceux dont le métier est d'éplucher des documents, c'est-à-dire

\section{La date du 18 correspond au calendrier russe.}


les historiens, et, à leur suite, par des hommes politiques qui auraient dû savoir la vérité ou qui la savaient. Pour ne prendre que cet exemple, le président du Conseil français du début de la guerre, René Viviani, faisait encore état que l'Autriche avait été la première à lancer son ordre de mobilisation générale dans un discours à la Chambre des députés le... 5 juillet 1922.

Un exemple remarquable est celui de Jules Isaac. En 1915, deux historiens qu'il considérait comme « ses maîtres », Émile Durkheim et Ernest Denis, écrivaient dans Qui a voulu la guerre?:

Ce fait capital que la mobilisation générale autrichienne a été antérieure à la mobilisation générale russe n'est, nulle part, signalé dans le Livre Blanc [allemand]. Cette antériorité est pourtant certaine. ${ }^{10}$

Jules Isaac avait d'abord accepté d'ajouter à l'ouvrage destiné à la classe de PhilosophieMathématiques, ce qu'aujourd'hui on appellerait terminale, un gros chapitre sur les origines de la guerre; il y reprenait évidemment cette thèse. Dans l'avant-propos de son livre sur les origines de la guerre, il commentait son comportement d'alors: «Aucun doute ne m'effleurait l'esprit. Je ne discutais pas, je détenais une certitude ${ }^{11}$. » Dès 1922, il rectifiait cette affirmation et s'en excusait; il devait récrire complètement son chapitre sur les origines de la guerre en 1929-1930.
Mais pendant les années vingt, livres d'histoire ou manuels destinés à l'enseignement secondaire reprenaient à peu près tous cette contre-vérité en s'appuyant sur les dires du Livre jaune.

Ces dires n'ont eu aucun effet sur le déroulement des événements pendant la guerre: tous les Français étaient convaincus de l'agression sans avoir eu besoin de le lire, conviction au demeurant justifiée. Que les Allemands aient cru impossible de ne pas appliquer le plan Schlieffen n'était pas un argument susceptible de la modifier!

Les affirmations contenues dans le Livre jaune ont en revanche servi de support, comme nous l'avons déjà indiqué, à certaines dispositions des traités de paix, fondées sur les responsabilités allemandes, en particulier le célèbre article 231 du traité de Versailles:

Les Gouvernements alliés et associés déclarent et l'Allemagne reconnaît que l'Allemagne et ses alliés sont responsables, pour les avoir causés, de toutes les pertes et de tous les dommages subis par les Gouvernements alliés et associés et leurs nationaux en conséquence de la guerre, qui leur a été imposée par l'agression de l'Allemagne et de ses alliés.

Cet article provoqua la fureur en Allemagne - colère telle que les Alliés purent se demander s'il se trouverait un gouvernement pour oser le signer. Il allait devenir le principal fondement

10. Émile Durkheim, Ernest Denis, Qui a voulu la guerre? Les origines de la guerre d'après les documents diplomatiques, A. Colin, 1915, p. 42 , n. 1 .

11. Jules Isaac, op. cit., p. 172, n. 2. 
d'un courant nationaliste allemand qui permit aux nazis de conquérir le pouvoir. La propagande nazie avait usé et abusé du thème de l'injustice faite aux Allemands. Ce n'est pas le moindre paradoxe que ce mensonge du Livre jaune eut peu d'importance pendant la Grande Guerre, mais qu'il contribua à la victoire nazie et à l'éclatement de la Deuxième Guerre mondiale!
En tout cas, quoi qu'en dise le Livre jaune, c'est bien la mobilisation générale russe qui a enclenché la guerre; sans elle, la guerre aurait peut-être eu lieu, tant les sentiments nationaux étaient portés à un haut niveau, mais peut-être pas, et la face du monde en aurait été changée. 